\title{
Suppression of the Pituitary-Adrenocortical System During Corticoid Therapy
}

\author{
Mitsuo NishiKaWA \\ The First Department of Medicine Niigata University \\ School of Medicine, Niigata, Japan
}

The excellent results of corticoid therapy have been widely recognized in various clinical conditions. However, suppressive effect of the pituitary-adrenocortical system is adherent to corticoids, from which even newer synthesized preparations can not be free. Many works have been published about this suppressive effect during administration of corticoids. The mechanism, however, has not been fully clarified. Our studies in this area have been done from the standpoint of the feed-back mechanism since several years ago. The main findings are summarized as follows.

Administration of prednisolone, dexamethasone or 6-methylprednisolone causes a decrease in urinary 17-OHCS day by day until around the 7 th day, when its excretion becomes minimal, in extreme cases to zero practically. This is already a well known fact and is utilized in differentiation of Cushing's syndromes as Jailer's test and in many experimental studies as hypophysectomy by drug.

In spite of receiving the same dosage of corticoids, however, their values then increase gradually until around the 14th day, when they may reach maximum to or over the pretreatment levels. Similar fluctuations can be seen after that and those levels generally become lower and lower.

In Fig. 1, each dot represents the mean value of 15 cases examined on the same day from the start of steroid administration and the curve is drawn from the mean values of every 4 days. These curves of urinary and plasma 17-OHCS show quite similar variations. (Blood were taken at 10 a.m. every day.)

As shown in Fig. 2, temporary rises in adrenal secretion occur soon after discontinuance of corticosteroid administration. The 


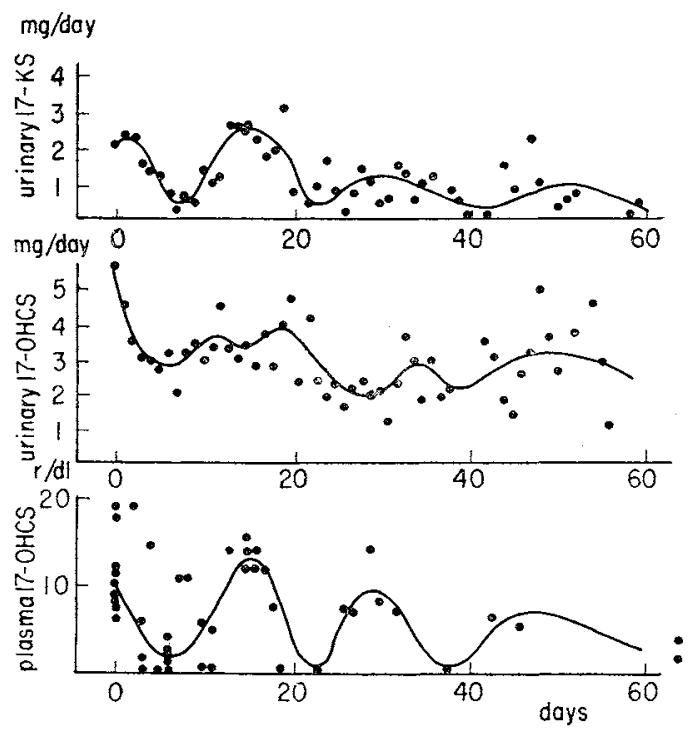

Fig. 1. Changes of urinary 17-OHCS, urinary $17-\mathrm{KS}$ and plasma 17-OHCS levels during corticosteroids therapy.

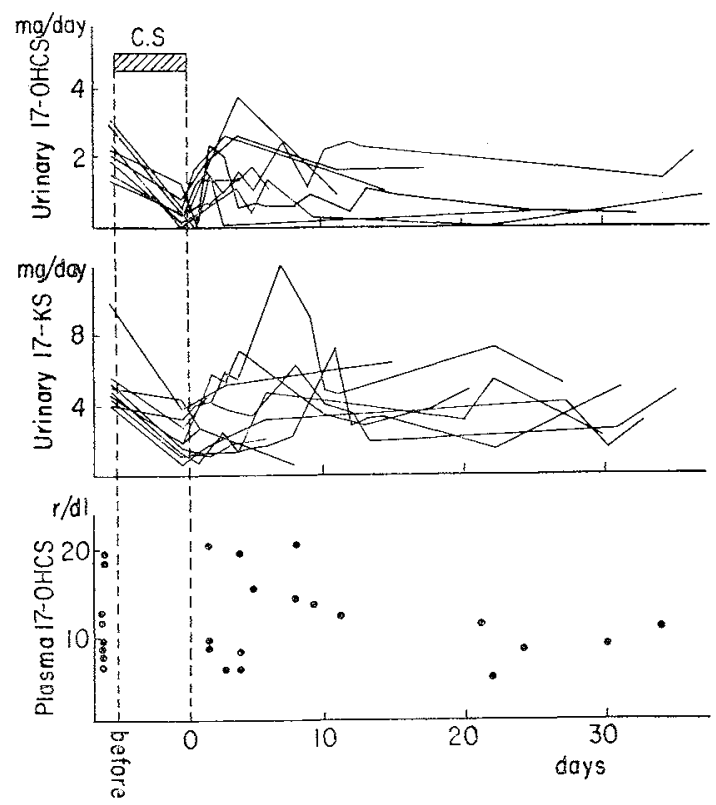

Fig. 2. Changes of urinary 17-OHCS, 17-KS and plasma 17-OHCS levels after corticosteroids therapy. 
maximal rises are found on the 3rd to 6 th day. After this, however, low levels under normal range are maintained for a period of over 30 days. Plasma concentration of 17-OHCS show also similar changes.

ACTH excreted in urine was repeatedly measured during corticoid therapy, because the suppression of the pituitary-adrenal system is considered to be caused chiefly by the disturbed secretion of ACTH. In spite of considerable variations day to day, tendencies can be seen that the urinary ACTH decreases soon after the start of corticoid administration, followed by the increase again from the 7 th or 8th day, which are quite parallel to the variations of 17-OHCS.

A similar relationship is found also when corticoid therapy is stopped. ACTH in urine is very small directly after the discontinuance, but on the $3 r d$ to 6 th day it increases rapidly.

After the 8th day, ACTH is not measured but it may be supposed that ACTH will sustain a low level for considerably long period, by reference to the finding of $17-$ OHCS.

The author summarized the above findins in fig. 3 . It is a very

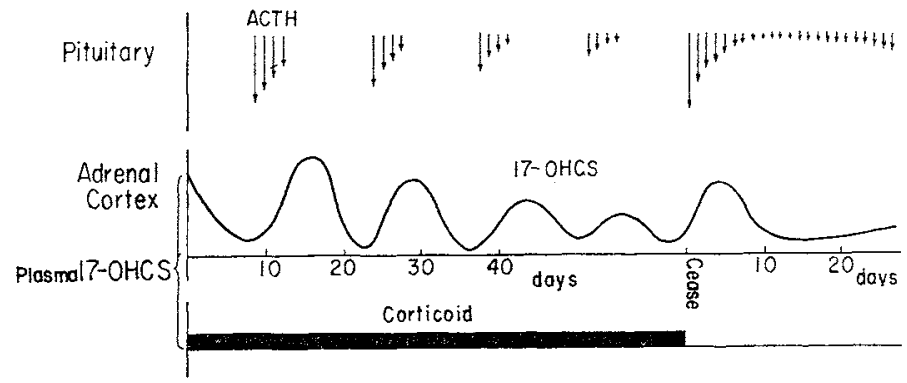

Fig. 3. Suppression of the pituitary adrenocortical system due to corticosceroids therapy.

interesting fact that the suppression does not last evently throughout the corticoid administration, but shows fluctuations with a period of about two weeks in spite of receiving the same dosage (3-6 tablets a day) and ACTH is released intermittently from the pituitary. In this figure, the fluctuating plasma concentration of 17-OHCS may be regarded as the endogenous one secreted by the adrenals of patients and the black bar as the exogenous one derived from the administered corticoid. 
Suppressive power against release of ACTH from the pituitary is the sum of the endogenous and the exogenous 17-OHCS. The concentration of the endogenous 17-OHCS is pretty high soon after the start of corticoid administration and the sum of the both $17-$ OHCS is strong enough to suppress release of ACTH from the pituitary. When the release of $\mathrm{ACTH}$ is stopped, the endogenous $17-$ OHCS will decrease and the sum of the endogenous and the exogenous will become so weak to suppress the pituitary. (The exogenous one is constant because corticosteroids are given in the same dosage every day.)

ACTH will be secreted again from the pituitary, and the endogenous 17-OHCS will be followed by the increase again. In such way the endogenous 17-OHCS shows daily fluctuation and ACTH is secreted intermittently.

In cases where corticoid administration was in large dosage than usual (10-20 tablets a day), secretions of 17-OHCS as well as ACTH maintain the suppressed low levels without any rise during the second week. Because the concentration of the exogenous 17 OHCS is so high in these cases, ACTH will be suppressed by only this exogenous one, unrelated with the concentration of the endogenous 17-OHCS.

The greater the daily dosage of administered corticoids, the

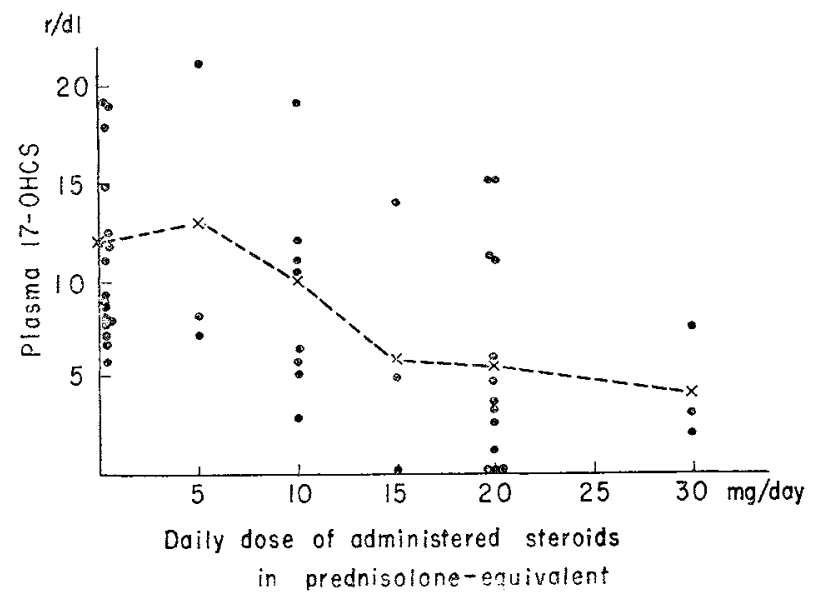

Fig. 4. Relationship of daily dose of administered corticoids to plasma levels of 17-OHCS. 
lower the plasma concentration of 17-OHCS as shown in Fig. 4. There is a daily variation in spite of administration of the same dosage of corticoids as described above and this figure shows that $5 \mathrm{mg}$. of steroid daily does not suppress the adrenal cortex, $10 \mathrm{mg}$. may suppress in some one and over $15 \mathrm{mg}$. daily does suppress in almost every one, to zero on some day. That is, the Japanese who are $50 \mathrm{~kg}$. of body weight in average, suffer from adrenal suppression by the administration of over $15 \mathrm{mg}$. of corticoids in terms of prednisolone, while if under $5 \mathrm{mg}$. is given very little reaction may be induced,

After discontinuance of the corticoid administration, temporary rises in 17-OHCS as well as ACTH appear on the $3 \mathrm{rd}$ to 6 th day and sustain subnormal level for long period after that. When the administration of corticoid is stopped, the exogenous 17-OHCS will decrease rapidly to zero and the endogenous one too, must be very low after long term corticosteroid therapy. Therefore the suppressive power against release of ACTH will become very weak suddenly and ACTH can be released from the pituitary, followed by the increase in 17-OHCS. The reason why these increases are only temporary, is a very interesting and important problem. The author believes that the amount of ACTH stored in the pituitary must be limited, and exhausted in a few days after disappearance of the suppressive effects, and further synthesis of ACTH must be lowered. That is, it is most reasonable, I think, that the administration of corticoids may suppress the release of ACTH from the pituitary as well as may lower the synthesis of ACTH, probably by way of suppression of the hypothalamic center.

The variations described above are the one when the administration of corticoid is stopped suddenly without lowering dosage. If we stop the administration after giving one tablet per day during about 2 weeks, temporary rises of 17-OHCS do not appear and pretty good reactions can be seen to SU tests which are carried out after 2 weeks after complete discontinuance of steroid administration. It is reasonable to give one tablet per day over 2 weeks at the end of corticoid therapy, because one tablet does not suppress the pituitary-adrenal system of patients and moreover replace the suppressed pituitary-adrenal system due to long term therapy. Of 
course there are some patients whose pituitary-adrenal systems do not react sufficiently even after such treatment and we must pay our special attention to such cases. 